arterial blood pressure. In addition, renalase appeared to decrease heart rate and cardiac contractility, without affecting peripheral vascular resistance.

The authors conclude that renalase is a novel, secreted flavin adenine dinucleotidedependent amine oxidase produced mainly by the kidney and involved in cardiovascular physiology. Further study of this protein might improve our understanding of the increased cardiovascular risk of patients with renal disease.

Original article Xu J et al. (2005) Renalase is a novel, soluble monoamine oxidase that regulates cardiac function and blood pressure. J Clin Invest [doi: 10.1172/JCI200524066]

\section{Evidence of a pharmacodynamic interaction between naproxen and low-dose aspirin}

Naproxen, a non-aspirin nonsteroidal antiinflammatory drug, has been investigated for its potential antithrombotic effect. Because results from clinical studies have been inconsistent, however, patients with cardiovascular disease and musculoskeletal disorders have tended to receive the drug in combination with aspirin. The value of this strategy has been questioned by a new study published in the Journal of the American College of Cardiology, which shows that naproxen might interfere with the cardioprotective effects of aspirin.

First, Capone and colleagues investigated the pharmacodynamic interaction between naproxen and aspirin in vitro using washed human platelets. Pretreatment of the platelets with naproxen was shown to reduce the irreversible inhibition of thromboxane $(\mathrm{TX}) \mathrm{B}_{2}$ production by aspirin, in a concentrationdependent manner.

Next, the investigators administered twicedaily, low-dose aspirin $2 \mathrm{~h}$ before naproxen (or vice versa) to four healthy volunteers for a period of 6 days. Ex vivo studies then showed that the inhibition of serum $\mathrm{TXB}_{2}$ production and platelet aggregation by aspirin did not appear to be affected by naproxen. A subsequent study in five volunteers showed, however, that concurrent administration of the two drugs resulted in no effects on platelet $\mathrm{TXB}_{2}$ production and aggregation at $1 \mathrm{~h}$ after dosing - the point at which aspirin alone causes maximal inhibitory effect. Moreover, only a transient inhibition of cyclooxygenase (COX)1 activity and function were detected. The rapid recovery of COX1 activity, say the authors, confirms that a pharmacodynamic interaction had occurred between aspirin and naproxen.

Capone et al. conclude that this interaction "might undermine the sustained inhibition of platelet COX1 that is necessary for aspirin's cardioprotective effects".

Original article Capone ML et al. (2005) Pharmacodynamic interaction of naproxen with low-dose aspirin in healthy subjects. J Am Coll Cardiol 45: 1295-1301

\section{Understanding early mortality after heart transplantation}

Levels of early mortality following cardiac transplantation have decreased in recent years, probably because of advances in the areas of donor/recipient selection, surgical techniques, immunosuppressive therapy and postoperative care. The cause of early death is unknown in many cases, however, and it is unclear whether further improvements are likely. Luckraz and colleagues have addressed these questions in their recent study.

The team reviewed the records of all 879 patients who underwent heart transplantation at their center from 1979 to June 2001. A total of 75 patients $(8.5 \%)$ died within 30 days of the operation. As expected, a sharp drop in 30-day mortality was observed between the 1979-1985 period (12.1\%) and the 19962001 period (6.9\%). Of the 75 early deaths, postmortem examination data indicated that almost one-third were due to graft failure. Acute rejection was responsible for more than one-fifth of deaths during the entire study period, but accounted for only $1.3 \%$ of cases between 1996 and 2001. Other major causes of death were sepsis (18.7\%), gastrointestinal problems $(9.3 \%)$ and postoperative bleeding (6.7\%). The remaining cases (12\%) died from a variety of causes including pulmonary embolism, pre-existing abdominal aortic aneurysm, and acute respiratory distress syndrome.

The investigators discuss opportunities for further improvements in early mortality by excluding borderline donors. Allografts 